\title{
A NOTE ON MAXIMAL OPERATORS AND REVERSIBLE WEAK TYPE INEQUALITIES
}

\author{
M. A. LECKBAND
}

\begin{abstract}
A class of maximal operators is shown to satisfy a weak type inequality and a corresponding converse inequality. The results are applicable to a fractionally iterated Hardy-Littlewood maximal operator.
\end{abstract}

1. In this paper we are interested in studying a class of maximal operators that satisfy a weak type inequality that is in some sense reversible. Let $M f$ denote the Hardy-Littlewood maximal operator of a function $f$ in $L^{\mathbf{1}}\left(\mathbf{R}^{n}\right)$. As is well known, for instance see [1], for suitable constants we have the weak type inequality

$$
\left|\left\{x \in \mathbf{R}^{n}: M f(x)>\lambda\right\}\right| \leq \frac{A_{n}}{\lambda} \int_{\left\{|f|>\lambda / A_{n}\right\}}|f(x)| d x
$$

and the converse inequality

$$
\frac{B_{n}}{\lambda} \int_{\left\{|f|>\lambda / B_{n}\right\}}|f(x)| d x \leq\left|\left\{x \in \mathbf{R}^{n}: M f(x)>\lambda\right\}\right| .
$$

Such a converse inequality naturally indicates how good the corresponding weak type inequality is. However, the motivation for the results in this paper is due to an application found in [4 and 5]. Knowing the weak type inequality and its converse for the Hardy-Littlewood maximal operator, an important formula for the $N$ th iteration of the maximal operator was obtained. That is, $M$ applied to $f N$ times is pointwise comparable to

$$
\sup _{x \in Q} \frac{1}{|Q|} \int_{0}^{|Q|}\left(f \cdot \chi_{Q}\right)^{*}(t) \frac{\log ^{N-1}}{(N-1) !}\left(\frac{|Q|}{t}\right) d t
$$

where $\left(f \cdot \chi_{Q}\right)^{*}$ is the nonincreasing rearrangement of $f$ restricted to the cube $Q$. As is shown in Theorem 3, this new operator satisfies a weak type inequality that has a converse. We replace $(1 /(N-1) !) \log ^{N-1}(|Q| / t)$ by a more general function and ask for weak type inequalities that have a converse result.

Let $\Phi:(1, \infty) \rightarrow[0, \infty)$ be measurable with $\int_{0}^{1} \Phi(1 / t) d t=1$. Let $f^{*}$ be the nonincreasing rearrangement of $f$, i.e.,

$$
f^{*}(t)=\inf \{s:|\{|f|>s\}| \leq t\} .
$$

We define the $\Phi$-maximal operator $M_{\Phi} f$ as

$$
M_{\Phi} f(x)=\sup \int_{0}^{1}\left(f \cdot \chi_{Q}\right)^{*}(|Q| t) \Phi\left(\frac{1}{t}\right) d t
$$

Received by the editors August 30, 1983.

1980 Mathematics Subject Classification. Primary 42B25. 
where the sup is extended over all cubes $Q$ with center $x$ and $\chi_{Q}$ is the characteristic function of $Q$.

The main results, Theorems 1 and 2, are proven for $\Phi$ which is either nonincreasing or nondecreasing, $t \Phi(t)$ nondecreasing, and for which there are positive constants $C_{1}, C_{2}, 0<\alpha<2,0<\beta<1$, such that $C_{1} t^{\alpha} \geq t \Phi(t) \geq C_{2} t^{\beta}$, for $1 \leq t<\infty$. Equivalent maximal operators where $\Phi(t)$ is nondecreasing have been studied (see [3]). For this reason perhaps the more interesting weak type estimates in Theorem 1 occur for $\Phi$ nonincreasing. Theorem 2 contains the converse inequalities to Theorem 1 . As a natural application, we consider the iterated Hardy-Littlewood maximal operator. Our results in Theorems 1 and 2 allow us to consider the case $\Phi(1 / t)=\log ^{a}(e / t)$, where $a$ is a given real number. We list the resulting corollaries as Theorem 3 .

2. Let $f: \mathbf{R}^{n} \rightarrow \mathbf{R}$ be a measurable function, let

$$
\lambda_{f}(s)=|\{x:|f(x)|>s\}|,
$$

and

$$
f^{*}(t)=\inf \left\{s: \lambda_{f}(s) \leq t\right\}
$$

For a reference on the properties of $f^{*}(t)$ see $[2]$. Let $\Phi:(1, \infty) \rightarrow[0, \infty)$ be a nonincreasing (nondecreasing), continuous function such that $\int_{0}^{1} \Phi(1 / t) d t=1$. We also require that $t \Phi(t)$ be nondecreasing and that there be positive constants $C_{1}, C_{2}, 0<\alpha<2$, and $0<\beta<1$ with $C_{1} t^{\alpha} \geq t \Phi(t) \geq C_{2} t^{\beta}$, for $1 \leq t<\infty$. We define the $\Phi$-maximal opertor of $f$ as

$$
M_{\Phi} f(x)=\sup _{Q} \int_{0}^{1}\left(f \cdot \chi_{Q}\right)^{*}(|Q| t) \Phi\left(\frac{1}{t}\right) d t
$$

where the sup is extended over all cubes $Q$ with center $x$, and $\chi_{Q}$ is the characteristic function of $Q$.

REMARK 1. If $f$ is not measurably constant, i.e., $|\{x:|f(x)|=a\}|=0$, for all $a>0$, then $M_{\Phi} f$ can be computed as

$$
M_{\Phi} f(y)=\sup \frac{1}{|Q|} \int_{Q}|f(x)| \Phi\left[\frac{|Q|}{\rho_{Q, f}(x)}\right] d x
$$

where the sup is extended over all cubes $Q$ with center $y$ and

$$
\rho_{Q, f}(x)=\inf \left\{t: x \in\left\{z:|f(z)| \geq\left(f \cdot \chi_{Q}\right)^{*}(t)\right\}\right\} .
$$

We use the convention that $A, B$, and $C$ denote constants depending only upon the dimension $n$ and $\Phi$. Any further dependence will be signified by a subscript.

The following lemma contains the inequalities that we will use to show the operator $M$ satisfies a weak type inequality.

LEMMA 1. Let $\lambda>0$ and $Q \subset \mathbf{R}^{n}$ such that

$$
\lambda \leq \int_{0}^{1}\left(f \cdot \chi_{Q}\right)^{*}(|Q| t) \Phi\left(\frac{1}{t}\right) d t .
$$

Then if $\Phi$ is nonincreasing we have

$$
1 \leq \frac{1}{|Q|} \int_{Q \cap\{|f|>\lambda / 2\}} C \frac{|f(x)|}{\lambda} \Phi\left[\left(C \frac{|f(x)|}{\lambda}\right)^{\beta}\right] d x .
$$


For $\Phi$ nondecreasing and $\eta>1 /(2-\alpha)$, there is a constant $C_{\eta}>0$ such that

$$
1 \leq \frac{1}{|Q|} \int_{Q \cap\{|f|>\lambda / 2\}} C_{\eta} \frac{|f(x)|}{\lambda} \Phi\left[\left(C_{\eta} \frac{|f(x)|}{\lambda}\right)^{\eta}\right] d x
$$

ProOF. Since $\Phi$ is normalized, that is $\int_{0}^{1} \Phi(1 / t) d t=1$, we have

$$
\frac{1}{2} \leq \int_{[0,1] \cap\left\{t:\left(f \cdot \chi_{Q}\right)^{*}(|Q| t)>\lambda / 2\right\}} \frac{\left(f \cdot \chi_{Q}\right)^{*}}{\lambda}(|Q| t) \Phi\left(\frac{1}{t}\right) d t .
$$

First we do the case where $\Phi$ is nondecreasing. For $0<\delta<1$ let

$$
J_{\delta}=\left\{t \in(0,1]: \frac{\left(f \cdot \chi_{Q}\right)^{*}}{\lambda}(|Q| t) \Phi\left(\frac{1}{t}\right) \leq \frac{1}{4} \frac{(1-\delta)}{t^{\delta}}\right\} .
$$

Then

or

$$
\frac{1}{2} \leq \int_{\left\{t:\left(f \cdot \chi_{Q}\right)^{*}(|Q| t)>\lambda / 2\right\} \backslash J_{\delta}} \frac{\left(f \cdot \chi_{Q}\right)^{*}(|Q| t)}{\lambda} \Phi\left(\frac{1}{t}\right) d t+\frac{1}{4}\left|J_{\delta}\right|,
$$

$$
\frac{1}{4} \leq \int_{\left\{t:\left(f \cdot \chi_{Q}\right)^{*}(|Q| t)>\lambda / 2\right\} \backslash J_{\delta}} \frac{\left(f \cdot \chi_{Q}\right)^{*}}{\lambda}(|Q| t) \Phi\left(\frac{1}{t}\right) d t .
$$

Let $t \in\left\{t:\left(f \cdot \chi_{Q}\right)^{*}(|Q| t)>\lambda / 2\right\} \backslash J_{\delta}$. We use the upper bound on $t \Phi(t)$ to estimate

$$
\frac{\left(f \cdot \chi_{Q}\right)^{*}(|Q| t)}{\lambda} \geq \frac{1-\delta}{4 \Phi(1 / t) t^{\delta}} \geq \frac{1-\delta}{4 C_{1}} t^{\alpha-(\delta+1)} .
$$

We choose $\delta$ such that $\alpha-(\delta+1)<0$, obtaining

$$
\left(C_{\eta} \frac{\left(f \cdot \chi_{Q}\right)^{*}(|Q| t)}{\lambda}\right)^{\eta} \geq \frac{1}{t}
$$

where

$$
\eta=\frac{1}{(\delta+1)-\alpha} \quad \text { and } \quad C_{\eta}=\max \left(4,\left(\frac{4 C_{1}}{1-\delta}\right)\right)
$$

Since $\Phi$ is nondecreasing we have

$$
\begin{aligned}
\frac{1}{4} & \leq \int_{\left\{t:\left(f \cdot \chi_{Q}\right)^{*}(|Q| t)>\lambda / 2\right\}} \frac{\left(f \cdot \chi_{Q}\right)^{*}(|Q| t)}{\lambda} \Phi\left[\left(C_{\eta} \frac{\left(f \cdot \chi_{Q}\right)^{*}(|Q| t)}{\lambda}\right)^{\eta}\right] d t \\
& =\frac{1}{|Q|} \int_{Q \cap\{|f|>\lambda / 2\}} \frac{|f(x)|}{\lambda} \Phi\left[\left(C_{\eta} \frac{|f(x)|}{\lambda}\right)^{\eta}\right] d x .
\end{aligned}
$$

Note that the continuity of $t \Phi\left[C_{\eta} t^{\eta}\right]$ gives us the above equality.

Now let $\Phi$ be nonincreasing. We may assume $C_{2}<\frac{1}{2}$, where $t \Phi(t)>C_{2} t^{\beta}$, and

$$
\int_{\left\{\left(f \cdot \chi_{Q}\right)^{*}(|Q| t)>\lambda / 2\right\}} \frac{\left(f \cdot \chi_{Q}\right)^{*}(|Q| t)}{\lambda} \Phi\left[\frac{\left(f \cdot \chi_{Q}\right)^{*}(|Q| t)}{C_{2}^{2 / \beta} \lambda}\right] d t<C_{2}^{2 / \beta} .
$$

If not, let $C=C_{2}^{-2 / \beta}$ and we are done, noting that $\beta<1$ and $\Phi$ is nondecreasing. With the above we use the lower bound for $t \Phi(t)$ and the property that $t \Phi(t)$ is nondecreasing to estimate

$$
\begin{aligned}
C_{2}\left[\frac{\left(f \cdot \chi_{Q}\right)^{*}(|Q| t)}{C_{2}^{2 / \beta} \lambda}\right]^{\beta} & \leq \frac{1}{t} \int_{0}^{t} \frac{\left(f \cdot \chi_{Q}\right)^{*}(|Q| s)}{C_{2}^{2 / \beta} \lambda} \Phi\left[\frac{\left(f \cdot \chi_{Q}\right)^{*}(|Q| s)}{C_{2}^{2 / \beta} \lambda}\right] d s \\
& \leq \frac{1}{t}
\end{aligned}
$$


provided $\left(f \cdot \chi_{Q}\right)^{*}(|Q| t)>\lambda / 2$. Thus,

$$
\begin{aligned}
\frac{1}{2} & \leq \int_{\left\{t:\left(f \cdot \chi_{Q}\right)^{*}(|Q| t)>\lambda / 2\right\}} \frac{\left(f \cdot \chi_{Q}\right)^{*}(|Q| t)}{\lambda} \Phi\left(\frac{1}{t}\right) d t \\
& \leq \int_{\left\{t:\left(f \cdot \chi_{Q}\right)^{*}(|Q| t)>\lambda / 2\right\}} \frac{\left(f \cdot \chi_{Q}\right)^{*}(|Q| t)}{\lambda} \Phi\left[\left(\frac{\left(f \cdot \chi_{Q}\right)^{*}(|Q| t)}{C_{2} \lambda}\right)^{\beta}\right] d t \\
& =\frac{1}{|Q|} \int_{Q \cap\{|f|>\lambda / 2\}} \frac{|f(x)|}{\lambda} \Phi\left[\left(\frac{|f(x)|}{C_{2} \lambda}\right)^{\beta}\right] d x
\end{aligned}
$$

This completes the proof of Lemma 1 .

Before continuing we would like to make an observation. If a specific nondecreasing $\Phi$ was under consideration, a better estimate could be obtained in Lemma 1 by using, for instance, $\left|t \log ^{2}[2 / t]\right|^{-1}$ instead of $t^{-\delta}$ in the proof. This would result in a sharper, though more complicated, inequality in Theorem 1. Not much is gained if $\Phi$ is a logarithmic function.

The next lemma contains the converse inequalities to Lemma 1 that will be used to prove Theorem 2. We follow convention and define

$$
\Phi^{+}(|f(x)|)=\Phi(\max \{1,|f(x)|\}) .
$$

LEMMA 2. Let $\lambda>0$ and $Q \subset \mathbf{R}^{n}$. If $\Phi$ is nonincreasing and $\eta>1 /(2-\alpha)$, then there is a $C_{\eta}$ such that

$$
|Q| \leq \int_{Q} \frac{|f(x)|}{\lambda} \Phi^{+}\left[\left(C_{\eta} \frac{|f(x)|}{\lambda}\right)^{\eta}\right] d x
$$

implies

$$
\frac{1}{4} \leq \int_{0}^{1} \frac{\left(f \cdot \chi_{Q}\right)^{*}}{\lambda}(|Q| t) \Phi\left(\frac{1}{t}\right) d t .
$$

If $\Phi$ is nondecreasing and

$$
|Q| \leq \int_{Q} \frac{|f(x)|}{\lambda} \Phi^{+}\left[\left(\frac{|f(x)|}{\lambda}\right)^{1 /(2-\beta)}\right] d x,
$$

then there is a constant $C$ such that

$$
1 \leq C \int_{0}^{1} \frac{\left(f \cdot \chi_{Q}\right)^{*}(|Q| t)}{\lambda} \Phi\left(\frac{1}{t}\right) d t .
$$

ProOF. We first assume $\Phi$ is nondecreasing. Let $B=\max \{1, \Phi(1)\}$ and $C_{2}$ be the constant, where $t \Phi(t) \geq C_{2} t^{\beta}, 1 \leq t<\infty$. We may assume

$$
\int_{0}^{1} \frac{\left(f \cdot \chi_{Q}\right)^{*}(|Q| t)}{\lambda} \Phi\left(\frac{1}{t}\right) d t<\frac{C_{2}}{2 B} .
$$

If not, we are done. Using the lower bound for $t \Phi(t)$ we estimate

$$
\begin{aligned}
\frac{\left(f \cdot \chi_{Q}\right)^{*}(|Q| t)}{\lambda} & \leq \frac{1}{t \Phi(1 / t)} \int_{0}^{t} \frac{\left(f \cdot \chi_{Q}\right)^{*}(|Q| s)}{\lambda} \Phi\left(\frac{1}{s}\right) d s \\
& \leq \frac{1}{2 B t^{2-\beta}} .
\end{aligned}
$$


Thus,

$$
1 \leq \int_{0}^{1} \frac{\left(f \cdot \chi_{Q}\right)^{*}(|Q| t)}{\lambda} \Phi^{+}\left[\left(\frac{\left(f \cdot \chi_{Q}\right)^{*}(|Q| t)}{\lambda}\right)^{1 /(2-\beta)}\right] d t
$$

implies

$$
\begin{aligned}
\frac{1}{2} & \leq \int_{\left\{t:\left(f \cdot \chi_{Q}\right)^{*}(|Q| t)>\lambda / 2 B\right\}} \frac{\left(f \cdot \chi_{Q}\right)^{*}(|Q| t)}{\lambda} \Phi\left[\left(2 B \frac{\left(f \cdot \chi_{Q}\right)^{*}(|Q| t)}{\lambda}\right)^{1 /(2-\beta)}\right] d t \\
& \leq \int_{0}^{1} \frac{\left(f \cdot \chi_{Q}\right)^{*}(|Q| t)}{\lambda} \Phi\left(\frac{1}{t}\right) d t .
\end{aligned}
$$

Now let $\Phi$ be nonincreasing. We require $C_{\eta} \geq 2 B$. Then

$$
1 \leq \int_{0}^{1} \frac{\left(f \cdot \chi_{Q}\right)^{*}(|Q| t)}{\lambda} \Phi^{+}\left[\left(C_{\eta} \frac{\left(f \cdot \chi_{Q}\right)^{*}(|Q| t)}{\lambda}\right)^{\eta}\right] d t
$$

implies

$$
\frac{1}{2} \leq \int_{\left\{t:\left(f \cdot \chi_{Q}\right)^{*}(|Q| t)>\lambda / 2 B\right\}} \frac{\left(f \cdot \chi_{Q}\right)^{*}(|Q| t)}{\lambda} \Phi\left[\left(C_{\eta} \frac{\left(f \cdot \chi_{Q}\right)^{*}(|Q| t)}{\lambda}\right)^{\eta}\right] d t .
$$

For $0<\delta<1$ let

$$
J_{\delta}=\left\{t \in(0,1]: \frac{\left(f \cdot \chi_{Q}\right)^{*}(|Q| t)}{\lambda} \Phi\left[\left(C_{\eta} \frac{\left(f \cdot \chi_{Q}\right)^{*}(|Q| t)}{\lambda}\right)^{\eta}\right] \leq \frac{(1-\delta)}{4 \cdot t^{\delta}}\right\} .
$$

Then

$$
\frac{1}{4} \leq \int_{\left\{t:\left(f \cdot \chi_{Q}\right)^{*}(|Q| t)>\lambda / 2 B\right\} \backslash J_{\delta}} \frac{\left(f \cdot \chi_{Q}\right)^{*}(|Q| t)}{\lambda} \Phi\left[\left(C_{\eta} \frac{\left(f \cdot \chi_{Q}\right)^{*}(|Q| t)}{\lambda}\right)^{\eta}\right] d t .
$$

For $t \in\left\{t:\left(f \cdot \chi_{Q}\right)^{*}(|Q| t)>\lambda / 2 B\right\} \backslash J_{\delta}$ we have, using $C_{1} t^{-\alpha} \geq t^{-1} \Phi\left(t^{-1}\right)$ and the above,

$$
\frac{C_{1}}{C_{\eta}}\left[C_{\eta} \frac{\left(f \cdot \chi_{Q}\right)^{*}(|Q| t)}{\lambda}\right]^{1-\eta+\alpha \eta} \geq \frac{1}{4} \frac{(1-\delta)}{t^{\delta}} .
$$

Hence, if we require $\eta /(1-\eta+\alpha \eta)>1$, i.e., $\eta>1 /(2-\alpha)$, we may choose $\delta=(1-\eta+\alpha \eta) / \eta<1$ and $C_{\eta}=\max \left\{2 B, 4 C_{1} /(1-\delta)\right\}$ to obtain

$$
\left(C_{\eta} \frac{\left(f \cdot \chi_{Q}\right)^{*}(|Q| t)}{\lambda}\right)^{\eta} \geq \frac{1}{t}
$$

Thus,

$$
\frac{1}{4} \leq \int_{0}^{1} \frac{\left(f \cdot \chi_{Q}\right)^{*}(|Q| t)}{\lambda} \Phi\left(\frac{1}{t}\right) d t
$$

and the proof of Lemma 2 is complete.

We now show the maximal $M_{\Phi} f$ satisfies a weak type inequality. The results are listed as Theorem 1.

THEOREM 1. Let $\lambda>0$. If $\Phi$ is nonincreasing there are constants $A$ and $C>2$ such that

$$
\left|\left\{x \in \mathbf{R}^{n}: M_{\Phi} f(x)>\lambda\right\}\right| \leq A \int_{\{|f|>\lambda / 2\}} \frac{|f(x)|}{\lambda} \Phi\left[\left(C \frac{|f(x)|}{\lambda}\right)^{\beta}\right] d x
$$


If $\Phi$ is nondecreasing there are constants $B_{\eta}$ and $C_{\eta}>2$ such that, for $\eta>1 /(2-\alpha)$,

$$
\left|\left\{x \in \mathbf{R}^{n}: M_{\Phi} f(x)>\lambda\right\}\right| \leq B_{\eta} \int_{\{|f|>\lambda / 2\}} \frac{|f(x)|}{\lambda} \Phi\left[\left(C_{\eta} \frac{|f(x)|}{\lambda}\right)^{\eta}\right] d x .
$$

ProOF. We let

$$
M_{r} f(x)=\sup \int_{0}^{1}\left(f \cdot \chi_{Q}\right)^{*}(|Q| t) \Phi\left(\frac{1}{t}\right) d t
$$

where the sup is extended over all $Q$ with center $x$ and $\operatorname{diam} Q \leq r$. It suffices to prove the theorem for $M_{r} f$ and then let $r \rightarrow \infty$.

Let $E_{\lambda}=\left\{x: M_{r} f(x)>\lambda\right\}$ and $E_{\lambda, R}=E_{\lambda} \cap\{|x|<R\}$. For $x \in E_{\lambda, R}$, we have a cube $Q_{x}$, with center $x$, and $\operatorname{diam} Q_{x} \leq r$ such that

$$
\lambda \leq \int_{0}^{1}\left(f \cdot \chi_{Q}\right)^{*}(|Q| t) \Phi\left(\frac{1}{t}\right) d t .
$$

We can now apply the Besicovitch covering theorem $[\mathbf{1}]$ and select $\left\{Q_{j}\right\} \subset\left\{Q_{x}: x \in\right.$ $\left.E_{\lambda, R}\right\}$ such that $E_{\lambda, R} \subset \bigcup Q_{j}$ and $\sum \chi_{Q_{j}}<C$, where $C$ depends only upon $n$. If we now assume $\Phi$ is nondecreasing, then by Lemma 1 we have

$$
\begin{aligned}
\left|E_{\lambda, R}\right| & \leq \sum\left|Q_{j}\right| \\
& \leq \sum C_{\eta} \int_{Q_{j} \cap\{|f|>\lambda / 2\}} \frac{|f(x)|}{\lambda} \Phi\left[\left(C_{\eta} \frac{|f(x)|}{\lambda}\right)^{\eta}\right] d x \quad\left(\eta>\frac{1}{2-\alpha}\right) \\
& \leq B_{\eta} \int_{\{|f|>\lambda / 2\}} \frac{|f(x)|}{\lambda} \Phi\left[\left(C_{\eta} \frac{|f(x)|}{\lambda}\right)^{\eta}\right] d x .
\end{aligned}
$$

We now let $R \nearrow \infty$ and then $r \nearrow \infty$ to complete the proof. The case where $\Phi$ is nondecreasing uses Lemma 1 and the proof is the same.

We now show that the maximal operator $M$ satisfies an inequality that is related to the converse of the corresponding weak type result of Theorem 1 .

THEOREM 2. Let $\lambda>0$. If $\Phi$ is nonincreasing we have, for some constants $A$ and $C_{\eta}$,

$$
\left|\left\{x \in \mathbf{R}^{n}: M_{\Phi} f(x)>\lambda A\right\}\right| \geq A \int_{\{|f|>\lambda / A\}} \frac{|f(x)|}{\lambda} \Phi^{+}\left[\left(C_{\eta} \frac{|f(x)|}{\lambda}\right)^{\eta}\right] d x
$$

where $\eta>1 /(2-\alpha)$.

If $\Phi$ is nondecreasing we have, for some constant $B$,

$$
\left|\left\{x \in \mathbf{R}^{n}: M_{\Phi} f(x)>\lambda B\right\}\right| \geq B \int_{\{|f|>\lambda / B\}} \frac{|f(x)|}{\lambda} \Phi^{+}\left[\left(\frac{|f(x)|}{\lambda}\right)^{1 /(2-\beta)}\right] d x .
$$

PROOF. We will just do the nonincreasing case, noting that the nondecreasing case is similar. For each of notation we let $\Phi_{1}(t)=\Phi\left[\left(C_{\eta} t\right)^{\eta}\right]$ for a fixed $\eta>$ $1 /(2-\alpha)$. Let

$$
\bar{f}(x)=\min \left\{r,|f(x)| \cdot \chi_{B(0, r)}(x)\right\} .
$$

Since $M_{\Phi} f(x) \geq M_{\Phi} \bar{f}(x)$, it suffices to prove the theorem for $\bar{f}(x)$ and then let $r \nearrow \infty$. 
We apply the Calderon-Zygmund Lemma $[6]$ to decompose $\mathbf{R}^{n}$ into sets $E$ and $F$ where

(i) $F \cap E=\varnothing$,

(ii) $(|\bar{f}(x)| / \lambda) \Phi_{1}^{+}(|\bar{f}(x)| / \lambda) \leq 2^{n}$, a.e. $x \in F$,

(iii) $E=\bigcup Q_{j}$, where $Q_{j} \cap Q_{k}=\varnothing$ for $j \neq k$ and

$$
2^{n} \leq \frac{1}{\left|Q_{j}\right|} \int_{Q_{j}} \frac{|\bar{f}(x)|}{\lambda} \Phi_{1}^{+}\left(\frac{|\bar{f}(x)|}{\lambda}\right) d x \leq 2^{2 n} .
$$

We note that (ii) implies $|\bar{f}(x)| \leq 2^{n} \lambda / \Phi(1)$ for a.e. $x \in F$. For a fixed $x \in Q_{j}$ let $Q_{x}$ be the smallest cube centered at $x$ containing $Q_{j}$. Then the above implies

$$
1 \leq \frac{1}{\left|Q_{x}\right|} \int_{Q_{x}} \frac{|\bar{f}(x)|}{\lambda} \Phi_{1}^{+}\left(\frac{|\bar{f}(x)|}{\lambda}\right) d x .
$$

Hence, by Lemma 2 , we have $M_{\Phi} \bar{f}(x) \geq \lambda / 4$ for every $x \in Q_{j}$. Summing over $Q_{j}$ and letting $A=\min \left\{2^{-2 n}, 2^{-n} \Phi(1)\right\}$ completes the proof.

3. Natural and interesting examples arise when $\Phi$ is a logarithmic type of function. In such a case Theorems 1 and 2 assume an elegant form since the exponents do not play a significant role.

Consider the following formula for the $N$ th iteration of the Hardy-Littlewood maximal operator (see [5, p. 5]):

$$
\underbrace{M \cdots M}_{N \text { times }} f(x) \sim \sup _{x \in Q} \int_{0}^{1}\left(f \cdot \chi_{Q}\right)^{*}(|Q| t) \frac{\log ^{N-1}(1 / t)}{(N-1) !} d t .
$$

We generalize the above formula for $-\infty<a<\infty$ as follows. For $a$ real let

$$
M_{a} f(x)=\sup _{x \in Q} \int_{0}^{1}\left(f \cdot \chi_{Q}\right)^{*}(|Q| t) \frac{\log ^{a}(e / t)}{C(a)} d t
$$

where $C(a)=\int_{0}^{1} \log ^{a}(e / t) d t$. We note that $e / t$ avoids the strong singularity at $t=1$ if $a \leq-1$. For this maximal operator, Theorems 1 and 2 assume a recognizable form which we state as Theorem 3 .

THEOREM 3. Let $\lambda>0$ and $a$ be a given fixed number. Then there exist constants $A$ and $B$ which depend only on dimension $N$ and a such that

$$
\begin{aligned}
& \left|\left\{x \in \mathbf{R}^{n}: M_{a} f(x)>\lambda\right\}\right| \leq A \int_{\{|f|>\lambda / A\}} \frac{|f(x)|}{\lambda}\left[1+\log ^{+}\left(\frac{|f(x)|}{\lambda}\right)\right]^{a} d x, \\
& \left|\left\{x \in \mathbf{R}^{n}: M_{a} f(x)<\lambda\right\}\right| \geq B \int_{\{|f|>\lambda / B\}} \frac{|f(x)|}{\lambda}\left[1+\log ^{+}\left(\frac{|f(x)|}{\lambda}\right)\right]^{a} d x .
\end{aligned}
$$

\section{REFERENCES}

1. M. de Guzmán, Differentiation of integrals in $\mathbf{R}^{n}$, Lecture Notes in Math., vol. 481, SpringerVerlag, New York, 1975.

2. R. A. Hunt, On $L(p, q)$-spaces, Enseign. Math. 12 (1966), 249-275.

3. W. B. Jurkat and J. L. Troutman, Maximal inequalities related to a.e. continuity, Trans. Amer. Math. Soc. 252 (1979), 49-64.

4. M. A. Leckband and C. J. Neugebauer, A general maximal operator and the $A_{p}$-condition, Trans. Amer. Math. Soc. 275 (1983), 821-831. 
5. __ Weighted iterates and variants of the Hardy-Littlewood maximal operator, Trans. Amer. Math. Soc. 279 (1983), 51-61.

6. E. M. Stein, Singular integrals and differentiability properties of functions, Princeton Univ. Press, Princeton, N.J., 1970.

DePARTMENT OF MATHEMATICS, Florida INTERNATIONAL UNiVERSity, Miami, FLORIDA 33199 